\title{
Fecal Occult Blood
}

National Cancer Institute

\section{Source}

National Cancer Institute. Fecal Occult Blood. NCI Thesaurus. Code C113708.

Blood in the feces that is not visibly apparent. 